\title{
SMOOTH GROUP ACTIONS ON DEFINITE 4-MANIFOLDS AND MODULI SPACES
}

\author{
IAN HAMBLETON AND RONNIE LEE
}

In this paper we give an application of equivariant moduli spaces to the study of smooth group actions on certain 4-manifolds. A rich source of examples for such actions is the collection of algebraic surfaces (compact and nonsingular) together with their groups of algebraic automorphisms. From this collection, further examples of smooth but generally nonalgebraic actions can be constructed by an equivariant connected sum along an orbit of isolated points. Given a smooth oriented 4-manifold $X$ which is diffeomorphic to a connected sum of algebraic surfaces, we can ask: (i) which (finite) groups can act smoothly on $X$ preserving the orientation, and (ii) how closely does a smooth action on $X$ resemble some equivariant connected sum of algebraic actions on the algebraic surface factors of $X$ ?

For the purposes of this paper we will restrict our attention to the simplest case, namely $X=P^{2}(\mathbf{C}) \# \cdots \# P^{2}(\mathbf{C})$, a connected sum of $n$ copies of the complex projective plane (arranged so that $X$ is simply connected). Furthermore,

Assumption. All actions will be assumed to induce the identity on $H_{*}(X, \mathbf{Z})$.

In previous works [17], [18], [19], we considered problem (i) and a variant of problem (ii) when $X=P^{2}(\mathrm{C})$. It turned out that the only finite groups which could act as above on $P^{2}(\mathbf{C})$ were the subgroups of $P G L_{3}(\mathbf{C})$ ([18] and [23] independently). For problem (ii) there are 2 interesting notions weaker than smooth equivalence. If $(X, \pi)$ is a smooth action, then the isotropy group $\pi_{x}=$ $\{g \in \pi \mid g x=x\}, x \in X$, acts linearly on the tangent space $T_{x} X$ and we can ask the following.

Question (iii) a. Given an action $(X, \pi)$, is there an equivariant connected sum of actions on $P^{2}(C)$ with the same fixed point data and tangential isotropy representations?

Question (iii) $b$. Given an action $(X, \pi)$, is there an equivariant connected sum of actions on $P^{2}(\mathbf{C})$ which is $\pi$-homotopy equivalent or $\pi$-equivariantly homeomorphic to $(X, \pi)$ ?

Partial results were obtained on these questions in [17] and [10]: if $\pi$ acts smoothly on $P^{2}(\mathbf{C})$, inducing the identity on homology, and the action has an

Received 19 January 1994.

Hambleton partially supported by NSERC grant A4000.

Lee partially supported by the NSF. 
isolated fixed point, then $\left(P^{2}(\mathbf{C}), \pi\right)$ is equivariantly homeomorphic to a linear action. We observed in [17] that this is false in general for actions of noncyclic groups $\pi$ on $P^{2}(\mathbf{C})$, due to the existence of actions with knotted 2-spheres in the singular set. There are related results for topological actions of cyclic groups on more general 4-manifolds in [9], [11], which will be summarized in $\S 1, \S 2$.

For the remainder of this paper, we will assume that $X=\#_{1}^{n} P^{2}(\mathbf{C})$ and concentrate on the case $n>1$. Our main results are as follows.

THeOREM A. Let $\pi$ be a finite group acting smoothly on $X=\#_{1}^{n} P^{2}(\mathbf{C})$. If $\pi$ induces the identity on homology, then $\pi$ is isomorphic to a subgroup of $P G L_{3}(\mathbf{C})$. If in addition $n>1$, then $\pi$ is abelian of rank $\leqslant 2$.

In the statement of the next result, we will let $\left[F_{1}\right],\left[F_{2}\right], \ldots,\left[F_{r}\right]$ denote the homology classes in $X$ of the oriented 2-dimensional components of the fixedpoint set, denoted by $\operatorname{Fix}(X, \pi)$, and $e_{1}, e_{2}, \ldots, e_{n}$ the standard basis of $H_{2}(X, Z)$ given by the complex embeddings $P^{1}(\mathbf{C}) \subset P^{2}(\mathrm{C})$ in each factor of the connected sum.

THEOREM B. Let $\pi=\mathbf{Z} / p$ be a cyclic group of prime order acting smoothly on $X=\#_{1}^{n} P^{2}(\mathbf{C})$, inducing the identity on homology. Then the given orientation on $X$ induces an orientation on $\operatorname{Fix}(X, \pi)$. Furthermore, there exist disjoint, nonempty subsets $S_{1}, S_{2}, \ldots, S_{r}$ of the set $\{1,2, \ldots, n\}$ such that $\left[F_{i}\right]=\sum\left\{e_{k} \mid k \in S_{i}\right\}$ for $1 \leqslant i \leqslant r$. If $p=2$, the subsets $S_{1}, S_{2}, \ldots, S_{r}$ form a partition of the set $\{1,2, \ldots, n\}$.

Here is a partial answer to Question (iii) a (see also [15], [16]).

THeOREM C. Let $(X, \pi)$ be a smooth cyclic group action on $X=\#_{1}^{n} P^{2}(\mathbf{C})$, inducing the identity on homology. Then there is an equivariant connected sum of actions of $P^{2}(\mathrm{C})$ with the same fixed-point data and tangential isotropy representations.

By combining Theorem $\mathrm{C}$ with the results of Edmonds and Ewing [11], we obtain the following.

THEOREM D. There exist locally-linear topological group actions $(X, \pi)$ of cyclic groups on connected sums of $P^{2}(\mathbf{C})$, inducing the identity on homology, which are not smoothable.

Before giving the proofs, we will use standard methods to collect some preliminary results about group actions on 4-manifolds. These include (\$1) P. A. Smith theory, and (\$2) the Atiyah-Singer index formula. Our new ingredient is in (\$3), namely equivariant gauge theory [19].

1. P. A. Smith theory. In this section we recall some results of A. Edmonds [9] and G. Bredon [4]. For convenience we have included proofs which apply to our special case. The idea is to study the Borel cohomology $H_{\pi}^{*}(X)=H^{*}\left(E \pi \times_{\pi} X, \mathrm{Z}\right)$ to obtain information about the singular set. It is easy to see that the spectral sequence for the fibration $X \rightarrow X \times{ }_{\pi} E \pi \rightarrow B \pi$ collapses [19, 6.1], [9, 2.3]. 
Corollary 1. Every class in $H^{2}(X, \mathbf{Z})$ is $c_{1}(L)$ for some $\pi$-equivariant $U(1)$ bundle $L \rightarrow X$.

Corollary 2. If $\pi=\mathbf{Z} / \mathbf{p}$ ( $p$ prime), then the fixed point set $F(X, \pi)$ is a disjoint union of isolated points and 2-spheres.

Proof. For $\pi=\mathbf{Z} / \mathbf{p}, X$ is totally nonhomologous to 0 in $X_{\pi}$, and it follows that

$$
\sum_{i} \operatorname{dim} H^{i}(X, \mathbf{Z} / \mathbf{p})=\sum_{i} \operatorname{dim} H^{i}(F, \mathbf{Z} / \mathbf{p})
$$

Since the action of $\pi$ is orientation-preserving, $F=F(X, \pi)$ is the disjoint union of $n_{P}$ isolated points $\left\{p_{i}\right\}$ and $n_{\mathrm{S}}$ surfaces $\left\{F_{k}\right\}$ of genus $\mathrm{g}\left(F_{k}\right)$. If $p$ is odd, the normal bundles to the surfaces $F_{k}$ have a natural orientation from the complex structure and so each $F_{k}$ is orientable. If $p=2$, we can use the fact that $H^{2 l}\left(X_{\pi}, \mathbf{Z} / 2\right) \cong$ $H^{2 l}\left(F_{\pi}, \mathrm{Z} / 2\right)$ for large $l$ to see that $\mathrm{Sq}^{1}$ is trivial on $H^{1}(F, \mathrm{Z} / 2)$, implying again that each surface $F_{k}$ is orientable (compare [4, page 379]).

Now the Euler characteristic

$$
\chi(F)=\sum_{k}\left(2-2 g\left(F_{k}\right)\right)+n_{P}=n+2
$$

and (*) implies that $\sum\left(2+2 g\left(F_{k}\right)\right)+n_{P}=n+2$, hence $g\left(F_{k}\right)=0$ for all $k$.

LEMMA 3. If $\pi=\mathbf{Z} / \mathbf{p}$, the map $H^{2}(\pi) \rightarrow H^{2}\left(\partial_{i}(X-N) / \pi\right)$ induced by the classifying map of the $\pi$-covering is injective for some component $\partial_{i}(X-N) / \pi$ of $\partial(X-N) / \pi$, where $N$ is a tubular neighbourhood of $F$ in $X$.

Proof. We have just seen that $2 n_{S}+n_{P}=n+2$. For $\pi=\mathbf{Z} / \mathbf{p}$ and $\mathbf{Z} / \mathbf{p}$ coefficients, there is an exact sequence

$$
0 \rightarrow H^{i}\left(X_{\pi}\right) \rightarrow H^{i}\left(F_{\pi}\right) \rightarrow H_{c p}^{i+1}((X-F) / \pi) \rightarrow 0
$$

Since $\operatorname{rank}_{\mathbf{Z} / \mathbf{p}} H^{2}\left(X_{\pi}\right)=n+1$, we get $H_{c p}^{3}((X-F) / \pi)=\mathbf{Z} / \mathbf{p}$. By Poincaré duality, $H_{1}((X-F) / \pi)=\mathbf{Z} / \mathbf{p}$, and the result is clear if $n_{P} \neq 0$ since the link of an isolated singular point in the quotient $X / \pi$ is a lens space.

If $n_{P}=0$, then $n_{S} \geqslant 2$ since we can assume $n>1$. Then we claim that each 2-sphere component $F_{i}$ of $F$ has a nontrivial normal bundle in $X$, implying that the link of $F_{i}$ in the quotient is a homology lens space, and we finish as before. If not, note first that suitable multiples (prime to $p$ ) of the circle fibres for the normal bundle in the quotient are homologous in $(X-F) / \pi$. Completing a 2-chain connecting these fibres by adjoining normal 2-disks in $N$ produces a 2-cycle in $X$ having a nontrivial intersection number with $F_{i}$. Therefore $0 \neq\left[F_{i}\right] \in H_{2}(X, Z)$. Since $X$ has definite intersection form, the Euler class of the normal bundle is $\left[F_{i}\right] \cdot\left[F_{i}\right] \neq 0$. 
Corollary 4. If $\pi=\mathbf{Z} / \mathbf{p}$, then the restriction map $H^{2}(X, \mathbf{Z} / \mathbf{p}) \rightarrow H^{2}(F, \mathbf{Z} / \mathbf{p})$ is surjective. Then 2-dimensional components of $F$ represent linearly independent elements in $\mathrm{H}_{2}(X, \mathbf{Z} / \mathbf{p})$.

Proof. Comparison of the spectral sequences for Borel cohomology of $F \subset X$ gives a commutative diagram

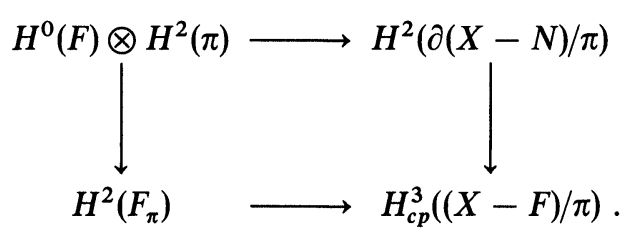

From Lemma 3 it follows that the composition

$$
H^{2}(\pi) \rightarrow H^{2}(\partial(X-N) / \pi) \stackrel{\delta}{\rightarrow} H_{c p}^{3}((X-F) / \pi)=\mathbf{Z} / \mathbf{p}
$$

is surjective (for $\mathbf{Z}$ or $\mathbf{Z} / \mathbf{p}$ coefficients) and a diagram chase finishes the proof:

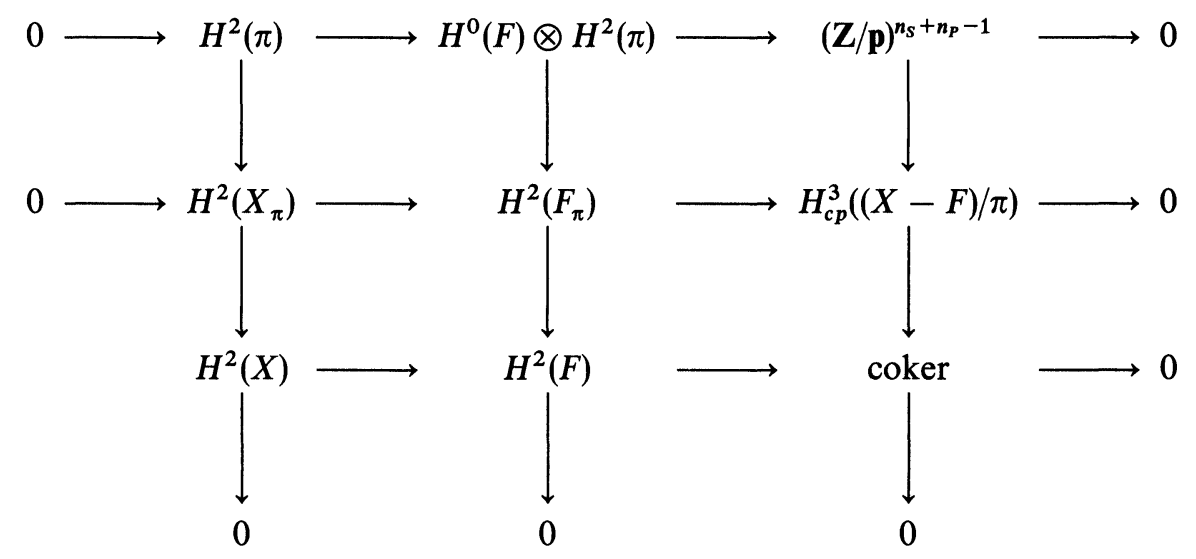

This result leads to the following question.

Question (iv). If $F_{i}$ is an oriented 2-sphere component of $F=\operatorname{Fix}(X, Z / \mathbf{p})$, what are the possibilities for $\left[F_{i}\right] \in H_{2}(X, Z)$ expressed in terms of the standard basis of $H_{2}(X, Z)$ given by the embeddings $P^{1}(\mathbf{C}) \subset P^{2}(\mathbf{C})$ in each factor?

Partial answers were provided by $[4,7.4]$ when $p=2$, and $[9,2.6]$ for any $p$, without assuming that the action induces the identity on homology or restricting the 4-manifold to be a connected sum of $P^{2}(\mathbf{C})$ 's. Note that in these results, the action is not necessarily smooth. In the smooth case, by applying gauge theory we obtain a complete answer to Question (iv) in the setting of Theorem B. 
2. Atiyah-Singer formulas. These formulas $[1, \S 6]$ relate the tangential isotropy representations of $g \in \pi$ to the equivariant signature, when $\pi$ acts locally-linearly on a 4-manifold $X$. If $p \in X$ is an isolated fixed point, and $t$ denotes a fixed 1-dimensional faithful complex representation of $\langle g\rangle$, then $T_{p}(X)=t^{a}+t^{b}$ for some integers $(a, b)$ relatively prime to $|g|$. If $F_{k}$ is a 2-sphere in $X$ fixed by $g$, then the normal bundle has the slice representation $\left.v\left(F_{k}\right)\right|_{x}=t^{\theta_{k}}, x \in F_{k}$.

(i) For $|g|$ odd,

$$
\operatorname{Sign}(g, X)=n=-\sum_{i} \cot \left(\frac{\mathrm{a}_{i}}{2}\right) \cot \left(\frac{b_{i}}{2}\right)+\sum_{k} \operatorname{cosec}^{2}\left(\frac{\theta_{k}}{2}\right) \cdot\left[F_{k}\right]^{2}
$$

where the sums correspond to the sets of isolated fixed points and fixed 2-spheres respectively.

(ii) For $|g|=2$,

$$
\operatorname{Sign}(g, X)=F \circ F \text {, }
$$

and in particular, isolated fixed points do not contribute.

A partial converse is provided by the following result of Edmonds and Ewing.

THEOREM 5 [11]. If $p$ is an (odd) regular prime, and the set of rotation numbers $\left\{\left(a_{i}, b_{i}\right)\right\}$ satisfies the Atiyah-Singer formula, then there exists a locally linear pseudofree topological $\mathbf{Z} / \mathbf{p}$-action on $X$ with these representations at the isolated fixed points.

In contrast, we will see in Theorem $\mathrm{D}$ that some of the actions constructed by Edmonds and Ewing are not smoothable, and so extra restrictions on the fixedpoint data exist for smooth actions.

3. Equivariant moduli spaces. In $[19, \S 2]$ we adapted the theory of Yang-Mills moduli spaces [7], [8] to the equivariant setting. Let $P \rightarrow X$ be a principal SU(2)bundle and suppose that $(X, \pi)$ is a smooth action with $\pi$ finite. We fix a real analytic structure on $X$ compatible with the $\pi$-action, and a real analytic equivariant metric. Then we constructed an equivariant perturbation of the Yang-Mills equations (using the method of Bierstone [2]) to construct a general position moduli space of self-dual connections $(\mathscr{M}, \pi)$ with an action of $\pi$. The main features are as follows.

(i) $(\mathscr{M}, \pi)$ is a Whitney stratified space [22] with an effective $\pi$-action and open manifold strata

$$
\mathscr{M}_{\left(\pi^{\prime}\right)}^{*}=\left\{x \in \mathscr{M}^{*} \mid \pi_{x}=\pi^{\prime} \subseteq \pi\right\}
$$

where $\mathscr{M}^{*} \subseteq \mathscr{M}$ is the subset of irreducible connections (up to gauge equivalence). 
(ii) For $\pi^{\prime} \subseteq \pi$ each component of the fixed point set $\mathscr{M}^{\pi^{\prime}}$ is the moduli space of $\tilde{\pi}^{\prime}$-invariant connections on $P$ with respect to a $\tilde{\pi}^{\prime}$-SU(2) bundle structure on $P$ (compare [14], [13]). Here $\tilde{\pi}^{\prime}$ is the preimage of $\pi^{\prime}$ under the surjection $\mathscr{G}(\pi) \rightarrow \pi$, where $\mathscr{G}(\pi)$ is the group of SU(2)-bundle automorphisms of $P$ which cover an isometry $g: X \rightarrow X$ given by the action of some element $g \in \pi$.

(iii) The strata have topologically locally trivial equivariant cone bundle neighbourhoods in $(\mathscr{M}, \pi)$.

(iv) $(\mathscr{M}, \pi)$ has an equivariant compactification $(\overline{\mathscr{M}}, \pi)$. When $c_{2}(P)=-1,(\overline{\mathscr{M}}, \pi)$ $=(\mathscr{M} \cup X \times[0,1), \pi)$, where $\tau: X \times 0 \subset \overline{\mathscr{M}}$ is the Taubes embedding of $X$ as the set of ideal "concentrated" connections and $X \times[0,1)$ is embedded as an equivariant collar of the given $\pi$-action on $X$.

(v) The dimensions of the strata $\mathscr{M}_{\left(\pi^{\prime}\right)}$ can be computed from the $\pi^{\prime}$-fixed set of the fundamental elliptic complex. A stratum $\mathscr{M}_{\left(\pi^{\prime}\right)}$ is nonempty only when its formal dimension is positive.

In [3], Bierstone gave a refinement of the definition of equivariant general position and showed that it is equivalent to a notion of equivariant stability or infinitesimal equivariant stability. We can modify our construction of equivariant moduli spaces to take this refinement into account. An advantage of this approach is that nearby perturbations result in smoothly equivalent stratified moduli spaces. Moreover, the internal structure of normal cone bundles along each stratum is now smoothly locally trivial.

We will consider the instanton number 1 moduli space (i.e., $c_{2}(P)=-1$ ) and discuss 4 additional features.

(a) Reducible connections. In general, reducible connections correspond to splittings $E=L \oplus L^{-1}$ of the associated $C^{2}$-bundle to $P$, as a sum of line bundles. In our case, there are $n>1$ reductions from line bundles $\left\{L_{i}\right\}$ with $c_{1}\left(L_{i}\right)=e_{i}$, $1 \leqslant i \leqslant n$, where $e_{i} \in H^{2}(X, Z)$ is the Poincaré dual of the $i$ th standard basis element. By Corollary 1 , we can assume that each $L_{i}$ is a $\pi-U(1)$ bundle over $X$, and so the associated reducible connection $\left[D_{i}\right]$ is fixed by the $\pi$-action on $\mathscr{M}$.

Next we consider neighbourhoods of a reducible connection $[D]$ in $\mathscr{M}$ and in $\mathscr{B}$. Recall that $[D]$ has a neighbourhood $\mathscr{U}^{*} \subset \mathscr{B}^{*}$ which is the weak homotopy type of $\mathbf{C} P^{\infty}[8,5.1 .18]$. We may assume that $\mathscr{U}^{*}$ is $\pi$-invariant, and then for $\pi=\mathbf{Z} / \mathbf{p}, p$ prime, it follows that the action $\left(\mathscr{U}^{*}, \mathbf{Z} / \mathbf{p}\right)$ is weakly $\mathbf{Z} / \mathbf{p}$-equivariantly homotopy equivalent to a linear action on $T_{D, \varepsilon}^{*} / S^{1}$ where $T_{D, \varepsilon} \subset \mathscr{A}$ is a transverse slice to the $\mathscr{G}$-orbit.

LEMMA 6. If $\pi=\mathbf{Z} / \mathbf{p}$, then $\operatorname{Fix}\left(\mathscr{U}^{*}, \mathbf{Z} / \mathbf{p}\right)$ is a disjoint union of $p$ subspaces $\mathscr{U}^{*}(\chi)$, each weakly homotopy equivalent to $\mathbf{C} P^{\infty}$, associated to the decomposition of $T_{D, \varepsilon}$ into complex linear subspaces $T_{D, \varepsilon}(\chi)$ on which $\mathbf{Z} / \mathbf{p}$ acts via a fixed character $\chi: \pi \rightarrow$ $S^{1}$. In addition, if $\mathscr{F}_{0}$ is a component of $\operatorname{Fix}\left(\mathscr{U}^{*}, \mathbf{Z} / \mathbf{p}\right)$, then the restriction map $H^{*}\left(\mathscr{U}^{*}, \mathbf{Z} / \mathbf{p}\right) \rightarrow H^{*}\left(\mathscr{F}_{0}, \mathbf{Z} / \mathbf{p}\right)$ is surjective.

Finally, we have the following result of Donaldson. 
Proposition $7[8,5.1 .21]$. For any $a \in H_{2}(X . \mathrm{Z})$, the restriction of $\mu(a)$ to the copy of $\mathbf{C} P^{\infty}$ which links the reducible connection $\left[D_{i}\right]$ is given by

$$
\left.\mu(a)\right|_{\mathbf{C P \infty}}=-\left\langle c_{1}\left(L_{i}\right), a\right\rangle \cdot h
$$

where $h \in H^{2}\left(\mathbf{C} P^{\infty}, \mathbf{Z}\right)$ is the positive generator, and $\mu: H_{2}(X, \mathbf{Z}) \rightarrow H^{2}\left(\mathscr{B}^{*}, \mathbf{Z}\right)$ is the map defined in $[8,5.1 .11]$. The composition

$$
H_{2}(X, \mathbf{Z}) \stackrel{\mu}{\rightarrow} H^{2}\left(\mathscr{B}^{*}, \mathbf{Z}\right) \stackrel{\tau^{*}}{\rightarrow} H^{2}(X, \mathbf{Z})
$$

is Poincaré duality [8, 5.3.3].

(b) Orientation. Donaldson shows that the real determinant line bundle $\Lambda(P)$ associated to the elliptic complex

$$
\Omega_{-}^{*}(\operatorname{Ad} \mathrm{P}): 0 \rightarrow \Omega^{0}(\operatorname{Ad} \mathrm{P}) \rightarrow \Omega^{1}(\operatorname{Ad} \mathrm{P}) \rightarrow \Omega_{-}^{2}(\operatorname{Ad} \mathrm{P}) \rightarrow 0
$$

has a canonical trivialization over $\mathscr{B}$ which induces the given orientation on $X$ times the inward pointing normal, where $X$ is embedded as the Taubes boundary in $\mathscr{M}[8, \S 5.4]$.

LeMMA 8. Let $\pi=\mathbf{Z} / \mathbf{p}$ for $p$ an odd prime, and $C \subset \mathscr{M}_{(\pi)}^{*}$ a connected component. Then $C$ is an orientable manifold.

Proof. If $[D] \in C$, we can split the elliptic complex

$$
\Omega_{-}^{*}(\operatorname{Ad~} \mathrm{P})=\Omega_{-}^{*}(\operatorname{Ad~} \mathrm{P})^{\pi} \oplus\left[\Omega_{-}^{*}(\operatorname{Ad} \mathrm{P})^{\pi}\right]^{\perp}
$$

into a fixed subcomplex and a perpendicular complex. It follows that the line bundle $\Lambda(P)=\Lambda_{t(C)} \otimes \Lambda_{n(C)}$, where $\Lambda_{t(C)}$ is the determinant line bundle of $\Omega_{-}^{*}(\operatorname{Ad~P})^{\pi}$ and $\Lambda_{n(C)}$ is for the complementary part of $\Omega_{-}^{*}(\operatorname{Ad} P)$. Since $\pi$ is odd-order cyclic, the action of $\pi$ on $\left[\Omega_{-}^{*}(\mathrm{Ad} \mathrm{P})^{\pi}\right]^{\perp}$ induces a complex structure and hence an orientation on $\Lambda_{n(C)}$. Then $\Lambda_{t(C)}$ is orientable also. However, the moduli space is locally modelled on the zero set of a smooth map $f: H_{D}^{1} \rightarrow H_{D}^{2}$ in Bierstone general position. Furthermore, the fixed set (coker $\left.d f_{0}\right)^{\pi}=0$ and ker $d f_{0}$ (which is fixed under $\pi$ ) is the tangent space to the manifold stratum $C$ at $[D]$. Therefore $C$ is orientable.

(c) Connectedness. Since our equivariant moduli space $(\mathscr{M}, \pi)$ is constructed by general position, it is unique only up to a suitable notion of cobordism. In particular, we can try to arrange for it to be as connected as possible.

LEMMA 9. For each reducible connection $[D]$, there exists an equivariant neighbourhood $\left(\mathscr{U}^{*}, \pi\right) \subset\left(\mathscr{B}^{*}, \pi\right)$ such that the closure of the free stratum in $\mathscr{M}^{*} \cap \mathscr{U}^{*}$ contains all singular strata of dimension $<5$. Moreover, for each singular point, the intersection of its link with the free stratum is connected. 
Proof. Let $\Sigma \subseteq \partial \mathscr{U} \cap \mathscr{M}^{*}$ be the link of $[D]$ in the closure of the free stratum of $(\mathscr{M}, \pi)$. We will identify $\mathscr{U} \cap \mathscr{M} \approx \phi^{-1}(0) / S^{1}$, where $\phi: H_{D}^{1} \rightarrow H_{D}^{2}$ is a local chart for the moduli space. Here the spaces $H_{D}^{i}$ are complex $\pi$ representations and $\phi$ is equivariant with respect to the $\pi \times S^{1}$ actions. Note that the $S^{1}$-action inducing the complex structure is free except at the origin. Then $\Sigma=\tilde{\Sigma} / S^{1}$ is the quotient space of the intersection $\tilde{\Sigma}$ of a sphere (of small radius) in $H_{D}^{1}$ with $\phi^{-1}(0)$.

By the index formula, the dimension of the free stratum equals 5 . For a singular stratum in $\phi^{-1}(0) / S^{1}$, its normal cone structure can be determined by applying Bierstone general position to $\phi: H_{D}^{1} \rightarrow H_{D}^{2}$ with respect to the isotropy subgroup $\pi^{\prime} \subset \pi$ of the stratum. From this it follows that the closure of the free stratum is the union of the free stratum with singular strata of dimension $<5$. It remains to show that the closure of the free stratum is connected in a neighbourhood of the reducible connection $[D]$.

Since the strata have even codimension, if $p, q$ are in distinct connected components of $\Sigma$, we may assume that $p$ lies in the free stratum and that there is an arc $\ell_{p q}$ joining $p$ and $q$, such that $\ell_{p q}(t)$ lies in the free stratum of $\Sigma$ for $0 \leqslant t<1$. Now we can assume that the image of the path $\ell(t)$ under $\phi$ is a simple closed path such that the interior points, $\ell(t)$ for $0<t<1$, lie in the free stratum of $\mathrm{H}_{D}^{2} / S^{1}$. Since the $\pi$-action $H_{D}^{1}$ is the cone over an action on the unit sphere, we can join $p$ and $q$ radially to the origin, and this closed path in $H_{D}^{1} / S^{1}$ bounds an embedded 2-disk $B$ whose interior lies in the free stratum. Then we deform $\phi=\phi_{0}$ in $H_{D}^{2}$ through general position maps $\phi_{s}, 0 \leqslant s \leqslant 1$, so that $\phi_{1}(B)=0$ and the new zero set $\phi_{1}^{-1}(0) / S^{1} \approx \phi_{0}^{-1}(0) / S^{1} \cup B$ in a neighbourhood of the origin. Note that the above construction can be made in the smooth category. Since a Bierstone general position map is determined by its jets up to some level $n_{0}$, i.e., by all its partial derivatives $\left(\partial / \partial x^{i_{1}} \cdots x^{i_{k}}\right) \phi$ of degree $\leqslant n_{0}$, it follows that the closure of the free stratum is connected.

(d) Domination and links of reducibles. After the last section we can assume that the link $\Sigma \subseteq \partial \mathscr{U} \cap \mathscr{M}^{*}$ of a reducible connection $[D]$ inside the closure of the free stratum is connected. In order to obtain information about its topology, we can compare our equivariant moduli space to the nearby generic moduli spaces without group action. By integrating a suitable vector field in a compact subset of a local chart, we can construct a continuous map $f: P^{2}(\mathbf{C}) \rightarrow \Sigma$ such that $f_{*}$ : $H_{4}\left(P^{2}(\mathbf{C}), \mathbf{Z}\right) \rightarrow H_{4}(\Sigma, Z)$ is degree 1 . Here $P^{2}(\mathbf{C})$ is the link of $[D]$ in a generic 5-dimensional smooth moduli space near $(\mathscr{M}, \pi)$, and $\Sigma$ carries a 4-dimensional class because the fixed-point sets have codimension at least 2 .

LEMMA 10. The link $\Sigma$ has the homotopy type of a 4-dimensional Poincaré complex.

Proof. By general position, the free stratum of $\Sigma$ is a manifold, but there could be nonmanifold points in the singular set. Let $p \in \Sigma$ be an isolated fixed point, with link $L=\ell k(p)$ in $\Sigma$. Since $p$ is isolated, the $\pi$ action on $\ell k(p)$ is free and $L$ is a connected 3-manifold by Lemma 9 . However, $L$ is dominated by a 
degree-1 map from $S^{3}$, and so $L$ is a homotopy 3-sphere and $\Sigma$ is a homology manifold around $p$. A similar argument deals with neighbourhoods of points which lie on 2-dimensional fixed sets.

Corollary 11. $\Sigma$ is homotopy equivalent to $P^{2}(\mathbf{C})$.

Proof. The Poincare duality space $\Sigma$ is the quotient space of a free $S^{1}$ action on $\tilde{\Sigma}$. But by the same domination argument, there is a degree-1 map $S^{5} \rightarrow \tilde{\Sigma}$. Therefore $\tilde{\Sigma}$ is homotopy equivalent to $S^{5}$ and $H_{2}(\Sigma)=\mathbf{Z}$. Now the existence of the degree-1 map $f: P^{2}(\mathbf{C}) \rightarrow \Sigma$ implies that $\Sigma$ has the integral homology of $P^{2}(\mathbf{C})$, and $\pi_{1}(\Sigma)=1$.

4. The proof of Theorem A (1). We will prove that our equivariant moduli space $(\mathscr{M}, \pi)$ has nice neighbourhoods around each reducible connection. First we need some preparation.

LEMMA 12. Let $C \subseteq \mathscr{M}_{\left(\pi^{\prime}\right)}^{*}$ be a noncompact connected component with $\pi^{\prime} \neq 1$. If $\operatorname{dim} C \geqslant 5$, then $C$ must be empty. If $\operatorname{dim} C=3$, then the closure $\bar{C} \subset \bar{M}$ must intersect the Taubes boundary $\partial \overline{\mathscr{M}}=X \times 0 \subset \bar{M}$.

Proof. If $\operatorname{dim} C \geqslant 5$, by general position (or more specifically the existence of normal cone bundle neighbourhoods to the strata) the closure $\bar{C} \subset \bar{M}$ does not intersect the closure of the free stratum except at reducible connections. If $\operatorname{dim} C=$ 3 , we will assume that $\bar{C}$ also has only reducibles as its limit points and derive a contradiction.

Choose a nontrivial subgroup $\mathbf{Z} / \mathbf{p} \subseteq \pi^{\prime}$, and put $(\mathscr{M}, \mathbf{Z} / \mathbf{p})$ in general position with respect to the action restricted to $\mathbf{Z} / \mathbf{p} \subset \pi$. Then the deformed stratum $C^{\prime}$ is a manifold stratum, fixed under $\mathbf{Z} / \mathbf{p}$, and $\overline{C^{\prime}}=C^{\prime} \cup$ \{reducibles $\}$. Let $\left[D_{1}\right], \ldots,\left[D_{r}\right] \in$ $\overline{C^{\prime}}$ be the set of reducible limit points of $\overline{C^{\prime}}$, and let $W$ denote the compact manifold with boundary obtained from $\overline{C^{\prime}}$ by removing small open neighbourhoods of the $\left[D_{i}\right]$. Then $W$ has odd dimension, say $2 d+1, d \geqslant 1$, and has 1 boundary component $\partial_{i} W \approx P^{d}(C)$ for each reducible $\left[D_{i}\right]$ in the closure of $C^{\prime}$.

Bierstone general position implies that the inclusion of each link $P^{d}(C) \subset \mathscr{B}^{*}$ induces a weak homotopy equivalence with the link of $\left[D_{i}\right]$ in $\mathscr{B}^{*}$ through dimensions $\leqslant 2 d$. If $\pm e_{i} \in H_{2}(X, Z)$ is the Poincaré dual of $c_{1}\left(L_{i}\right)$, where $L_{i}$ is the line bundle associated to the reduction, then

$$
\left.\mu\left(e_{i}\right)\right|_{\partial_{j} W}=-\left\langle c_{1}\left(L_{j}\right), e_{i}\right\rangle \cdot h_{j}= \pm \delta_{i j}
$$

where $h_{j} \in H^{2}\left(\partial_{j} W, \mathbf{Z}\right) \cong H^{2}\left(P^{d}(\mathbf{C}), \mathbf{Z}\right)$ is the positive generator. For any cocycle representative $\mu\left(\tilde{e}_{i}\right)$ of $\mu\left(e_{i}\right)$ we have

$$
\left.\left\langle\mu \tilde{e}_{i}\right)^{d},[\partial W]\right\rangle=\left\langle\delta\left(\mu\left(\tilde{e}_{i}\right)^{d}\right),[W]\right\rangle \equiv 0 \quad \bmod 2 .
$$

But since 


$$
\left\langle\mu\left(\tilde{e}_{i}\right)^{d},[\partial W]\right\rangle=\sum_{j=1}^{r}\left\langle\mu\left(e_{i}\right)^{d},\left[\partial_{j} W\right]\right\rangle=\left\langle h_{i}^{d},\left[P^{d}(\mathbf{C})\right]\right\rangle \equiv 1 \quad \bmod 2,
$$

we have a contradiction.

Recall that $\Sigma \subseteq \partial \mathscr{U} \cap \mathscr{M}^{*}$ denotes the link of a reducible connection [D] inside the closure of the free stratum. As in $\S 4$ (c), we identify $\mathscr{U} \approx H_{D}^{1} / S^{1}$, and $\mathscr{U} \cap \mathscr{M} \approx$ $\phi^{-1}(0) / S^{1}$, using a local chart map $\phi: H_{D}^{1} \rightarrow H_{D}^{2}$ in general position. Consider the decomposition of $V=H_{D}^{1}$ into isotypical $\pi$-subspaces $V=\bigoplus V\left(\chi_{i}\right)$, where each $V\left(\chi_{i}\right)$ is a direct sum of copies of the same irreducible complex representation of $\pi$. There is a similar decomposition for $W=H_{D}^{2}$, and we further write $V=V_{0} \oplus$ $V^{\prime}, W=W_{0} \oplus W^{\prime}$, where $V_{0}=\operatorname{ker} d f_{0}$ and $W_{0}=$ coker $d f_{0}$ are determined by the differential (at zero) of a general position map $f: V \rightarrow W$. We may assume $V_{0}$ and $W_{0}$ have no irreducible representations in common $[19,1.11]$. Let $\operatorname{deg}(\chi)$ denote the degree of a complex representation $\chi$, and $m_{0}(\chi)$ its multiplicity as a constituent of $V_{0}$.

COROLlaRY 13. If $\chi_{j}$ is an irreducible complex constitutent of $V_{0}$, then $m_{0}\left(\chi_{j}\right)$. $\operatorname{deg}\left(\chi_{j}\right) \leqslant 3$, with equality holding if and only if $\operatorname{deg}\left(\chi_{j}\right)=3, m_{0}\left(\chi_{j}\right)=1$, and $\chi_{j}$ is faithful as a projective representation of $\pi$ into $P G L_{3}(\mathbf{C})$.

Proof. If $\chi_{j}$ is an irreducible complex constitutent of $V_{0}$, then by general position there is a nonempty stratum component in $\mathscr{M}^{*}$ of (real) dimension $\geqslant 2 m_{0}\left(\chi_{j}\right) \operatorname{deg}\left(\chi_{j}\right)-1$ with minimal isotropy subgroup equal to the minimal isotropy subgroup occurring in a complex projective space of (real) dimension $2 d=2 \operatorname{deg}\left(\chi_{j}\right)-2$ on which $\pi$ acts via the associated projective representation of $\chi_{j}$ into $P G L_{d}(\mathbf{C})$.

If $\chi_{j}$ is a faithful projective representation of $\pi$, then its minimal isotropy group is the trivial group. Since every component of the free stratum of the moduli space has dimension 5 (from the usual Atiyah-Hitchin-Singer index calculation [19, page 28]), we conclude that $2 m_{0}\left(\chi_{j}\right) \operatorname{deg}\left(\chi_{j}\right)-1 \leqslant 5$. If equality holds, then the solution $m_{0}\left(\chi_{j}\right)=3$ and $\operatorname{deg}\left(\chi_{j}\right)=1$ would produce a 3 -dimensional projectively trivial subrepresentation of $V_{0}$, and hence an open subset of $\mathscr{M}^{*}$ on which $\pi$ acted trivially. This is ruled out by the effectiveness of the $\pi$-action on $\mathscr{M}$, which is a consequence of our assumption that the original equivariant metric on $X$ was chosen to be real analytic. (Recently [20], S. Illman has shown that every smooth compact group action admits an equivariant analytic structure.)

On the other hand, if $\chi_{j}$ is not faithful, its minimal isotropy group is the kernel $\pi^{\prime} \neq 1$ of the representation. Then, by Lemma 12 , any connected component of $\mathscr{M}_{\left(\pi^{\prime}\right)}^{*}$ has dimension $\leqslant 3$ and so $m_{0}\left(\chi_{j}\right) \cdot \operatorname{deg}\left(\chi_{j}\right) \leqslant 2$.

It will also be important for us to estimate how many singular strata intersect $\Sigma$. For each irreducible complex constituent $\chi_{j}$ of $V_{0}$, let $\Sigma\left(\chi_{j}\right)$ denote the intersection of $\Sigma$ with the complex projective subspace $\mathbb{P}\left(\chi_{j}\right)=S\left(V\left(\chi_{j}\right)\right) / S^{1} \subset \mathscr{U}$, and call this the singular stratum in $\Sigma$ associated to $V\left(\chi_{j}\right)$. Note that points $x \in \operatorname{Fix}(\Sigma, \pi)$ correspond to $\pi$-invariant 1-dimensional complex linear subspaces of $V_{0}$, so 
that these singular strata contain all the points of $\operatorname{Fix}(\Sigma, \pi)$. Let $d_{j}=m_{0}\left(\chi_{j}\right)$. $\operatorname{deg}\left(\chi_{j}\right)-1$.

LEMMA 14. There are at most 3 nonempty singular strata $\Sigma\left(\chi_{j}\right)$ of real dimension $2 d_{j}$ in the link $\Sigma$, and $\sum\left(d_{j}+1\right) \leqslant 3$ where we sum over all irreducible complex constituents $\chi_{j}$ of $V_{0}$.

Proof. We note that each nonempty $\Sigma\left(\chi_{j}\right)$ has the integral homology of a projective space, of real dimension $2 d_{j}$, where $0 \leqslant d_{j} \leqslant 2$ by Corollary 13. Moreover, if $d_{j} \geqslant 1$, then the inclusion induces an isomorphism $H_{2}\left(\Sigma\left(\chi_{j}\right), \mathbf{Z}\right) \rightarrow H_{2}(\Sigma, \mathbf{Z})$. The argument is similar to the proof of Corollary 11: each singular stratum is the orbit space of a simplicial complex homotopy equivalent to a sphere under a free $S^{1}$-action.

Since the different subspaces $\mathbb{P}\left(\chi_{j}\right)$ are all disjoint, so are the different $\Sigma\left(\chi_{j}\right)$. This implies that if $\Sigma\left(\chi_{j}\right) \neq \varnothing$ and $d_{j}=2$, then $\Sigma=\Sigma\left(\chi_{j}\right)$ has just 1 singular stratum.

Next, $d_{j}=1$ for some constitutent $\chi_{j}$ of $V_{0}$ would imply the existence of a $\pi$-invariant $S^{2}$ in $\Sigma$. But the nontrivial self-intersection number of the generator of $H_{2}(\Sigma, Z)$ implies that all other singular strata $\Sigma\left(\chi_{i}\right), i \neq j$ must have $d_{i}=0$.

We will finish the proof by considering 2 cases.

Case (i). Suppose that the $k+1$ nonempty singular strata all have $d_{j}=0$. In other words, $\operatorname{Fix}(\Sigma, \pi)$ consists of $k+1$ distinct points, and we need to show that $k \leqslant 2$. Let $Y=\Sigma-\left\{x_{1}\right\}$, where $x_{1} \in \Sigma$ denotes 1 of the fixed points.

Then $Y$ is a finite simplicial $\pi$-complex homotopy equivalent to $S^{2}$, and the $\pi$-action has $k$ fixed points corresponding to distinct (nontrivial) linear representations $\chi_{j}$ of $\pi$, each a complex constituent of $V_{0}$ with multiplicity 1 . Since the free stratum of $\Sigma$ is dense, the representation $V_{0}$ must be projectively faithful. For a sum of linear characters, this can only happen if $\pi$ is abelian.

Pick an element $1 \neq g \in \pi$ of prime order $p$. Then by the P. A. Smith theory, $\operatorname{Fix}(Y, g)$ is a $\mathbf{Z} / \mathbf{p}$-homology sphere containing $\operatorname{Fix}(Y, \pi)$. It follows that $\operatorname{Fix}(Y, g)$ is either an $S^{2}$ or consists of 2 distinct points. Since this fixed set is $\pi$-invariant, we obtain $k=2$ by an easy inductive argument.

Case (ii). Suppose that one of the singular strata, $\Sigma\left(\chi_{j}\right)$, has $d_{j}=1$, and the other $(k-1)$ strata have $d_{i}=0$ corresponding to distinct linear representations $\chi_{j}$ of $\pi$, each a complex constituent of $V_{0}$ with multiplicity 1 . Let $Y$ be obtained from $\Sigma$ by collapsing the invariant 2-sphere $\Sigma\left(\chi_{j}\right)$ to a point; then $Y$ is a finite simplicial $\pi$-complex homotopy equivalent to $S^{4}$, and the $\pi$ action has $k \geqslant 1$ distinct points in $\operatorname{Fix}(Y, \pi)$.

Consider a composition series $\pi_{0} \triangleleft \pi_{1} \triangleleft \cdots \triangleleft \pi_{r}=\pi$ for $\pi$, where each $\pi_{i} / \pi_{i-1}$ is simple or solvable. Assume by induction that $\operatorname{Fix}\left(Y, \pi_{i-1}\right)$ is either a 2-sphere or $\leqslant 2$ distinct points. If $\pi_{i} / \pi_{i-1}$ is solvable, then we show as in Case (i) that $\operatorname{Fix}\left(Y, \pi_{i}\right)$ is either a 2 -sphere or $\leqslant 2$ distinct points. If $\pi_{i} / \pi_{i-1}$ is simple, the trivial representation is the only representation of $\pi_{i} / \pi_{i-1}$ with degree 1 , and so $\operatorname{Fix}\left(Y, \pi_{i}\right)$ contains at most 2 points. 
The following result was proved for $\pi$ a cyclic 2-group in [5], [6], using other techniques.

THEOREM 15. Let $\pi$ act smoothly on $X$, inducing the identity on homology. Then for each reducible connection [D], there is a $\pi$-invariant neighbourhood of [D] in $(\mathscr{M}, \pi)$ which is smooth away from the cone point and $\pi$-homeomorphic to the cone of a linear action on $P^{2}(\mathbf{C})$. These 2 stratified spaces are equivariantly diffeomorphic away from the cone point.

Proof. By Lemma 12 we may now assume that $\operatorname{dim} \mathscr{M}_{\left(\pi^{\prime}\right)}^{*}<5$ for each stratum with nontrivial isotropy group $\pi^{\prime}$. Furthermore, by Lemma 10 within a local chart we can assume that there is a neighbourhood $\mathscr{U} \subset \mathscr{B}$ of each reducible [D] such that $\mathscr{U}^{*} \cap \mathscr{M}^{*}$ is connected. Our local model for $\mathscr{M}^{*}$ near $[D]$ is determined by a $\pi \times S^{1}$ equivariant map $f: H_{D}^{1} \rightarrow H_{D}^{2}$ in general position. As above, we write $V=H_{D}^{1}, W=H_{D}^{2}$ and decompose $V=V_{0} \oplus V^{\prime}, W=W_{0} \oplus W^{\prime}$, where $V_{0}=\operatorname{ker} d f_{0}$ and $W_{0}=$ coker $d f_{0}$.

By Lemma 14 there are at most 3 singular strata in the link $\Sigma$ of $[D]$ in $\mathscr{M}^{*}$. Moreover, $\sum\left(d_{j}+1\right) \leqslant 3$ implies that $V_{0} \subseteq V$ has at most 3 summands in its decomposition into isotypical components, and $\operatorname{dim} V_{0} \leqslant 6$. However, by the index calculation, $\operatorname{dim} H_{D}^{1}-\operatorname{dim} H_{D}^{2}=6$ so $W_{0} \subseteq W$ must be trivial, and it follows that $\mathscr{M}$ is locally modelled on a transverse equivariant map near each reducible.

Part 1 of Theorem A is now proved: the link of a reducible is the cone on some linear $\pi$-action on $P^{2}(C)$ and in particular, $\pi \subset P G L_{3}(\mathbf{C})$.

5. The proof of Theorem $A$ (2), and Theorem B. Suppose now that $X=$ $\#_{1}^{n} P^{2}(\mathrm{C})$ with $n \geqslant 2$. Theorem $\mathrm{B}$ is completed by Theorem 15 and the following result. Note that in the case of involutions $(p=2)$, the equivariant link $\left(P^{2}(\mathbf{C}), \pi\right)$ of each reducible contains a fixed 2-sphere $[18,2.1]$. This accounts for the last assertion in the statement of Theorem B.

THEOREM 16. Let $\pi=\mathbf{Z} / \mathbf{p}, p$ prime.

(i) Each 2-dimensional component $F_{i} \subset \operatorname{Fix}(X, \mathbf{Z} / \mathbf{p})$ has $\left[F_{i}\right]=\sum a_{i k} e_{k}$ where $a_{i k}$ $=0,1$ and (for each $i$ ) not all the $a_{i k}$ are 0 .

(ii) No nonempty collection $\left\{F_{i}\right\} \subset X=\partial \overline{\mathscr{M}}$ bounds a fixed set in $\mathscr{M}^{*} \cup \partial \overline{\mathscr{M}}$.

(iii) No nonempty collection of 2-dimensional fixed sets in the links of the reducible connections bounds a compact fixed set in $\mathscr{M}^{*}$.

Proof. Parts (i) and (ii) are proved by similar arguments to those above, using the $\mu$-map. Note that each noncompact 3-dimensional component of $\operatorname{Fix}\left(\mathscr{M}^{*}, \mathbf{Z} / \mathbf{p}\right)$ is canonically oriented since its closure in $\bar{M}$ meets $\partial \bar{M}=X$ and the set of reducibles. But the Donaldson orientation on $\mathscr{M}$ induces the standard orientation of $P^{2}(\mathbf{C})$ times the inward normal on the link $\Sigma=P^{2}(\mathbf{C})$ of each reducible, and the $\pi$-action on $\Sigma$ is complex linear, so the 2-dimensional fixed set in the links repre- 
sent the homology classes $P^{1}(\mathrm{C}) \subset P^{2}(\mathrm{C})$. The compatibility of this orientation with that of $X$ specifies the sign in the expression for $\left[F_{i}\right] \in H_{2}(X, Z)$. Part (iii) follows from Lemma 12.

The next result will be used in the proofs of Theorems $\mathrm{C}$ and $\mathrm{D}$.

LEMMA 17. If $\pi=\mathbf{Z} / \mathbf{p}, p$ an odd prime, then each noncompact 1-dimensional fixed set in $\mathscr{M}^{*}$ has at least 1 reducible connection as a limit point in $\mathscr{M}$.

Proof. Let $\gamma$ denote the closure in $\overline{\mathscr{M}}$ of a 1-dimensional fixed stratum of $\mathscr{M}^{*}$. Then if $\gamma$ contains no reducible connections, its endpoints $\left\{p_{1}, p_{2}\right\}$ lie in $X=\partial \overline{\mathscr{M}}$. Suppose that $\left(a_{1}, b_{1}\right)$ and $\left(a_{2}, b_{2}\right)$ denote the rotation numbers at $p_{1}$ and $p_{2}$ respectively. Then clearly $\left(a_{1}, b_{1}\right)$ and $\left(a_{2}, b_{2}\right)$ are a cancelling pair, or in other words we can assume that $\left(a_{2}, b_{2}\right)=\left(-a_{1}, b_{1}\right)$.

To rule out this pattern, we recall that a fixed-point component, such as the arc $\gamma$, corresponds to a $\pi$-equivariant structure on our original SU(2)-bundle $P$ over $X$ (compare [14]). Also recall that this bundle admits a concentrated connection at the point $p_{1}$ by means of Taubes construction. An equivariant interpretation of this last connection allows us to describe explicitly this $\pi$-bundle $(\pi, P)$ structure as follows.

Consider a small invariant 4-ball $B\left(p_{1}\right)$ around $p_{1}$. By collapsing the complement $X-B\left(p_{1}\right)$ of this 4-ball to a point, we obtain a 4-sphere $S^{4}=\mathbf{H} \cup\{\infty\}$ with a linear action and an equivariant, degree-1 map $g_{1}: X \rightarrow S^{4}$ sending $p_{1}$ to the north pole, $g_{1}\left(p_{1}\right)=0$, and sending the complement $X-B\left(p_{1}\right)$ to the south pole, $g_{1}\left(X-B\left(p_{1}\right)\right)=\infty$. Locally near the north pole, the group action is given by the rotation numbers $\left(a_{1}, b_{1}\right)$ and near the south pole by $\left(-a_{1}, b_{1}\right)$. As described in [21, Theorem 3], there exists an equivariant $\mathrm{SU}(2)$-bundle $Q$ over $S^{4}$ whose Chern number $c_{2}(Q)=-1$ and whose rotation number $\left(\pi,\left.Q\right|_{0}\right)$ at the north pole is $\left\{\left(a_{1}+b_{1}\right),-\left(a_{1}+b_{1}\right)\right\}$ and at the south pole is $\left\{\left(-a_{1}+b_{1}\right),-\left(-a_{1}+b_{1}\right)\right\}$. Pulling back $Q$ by means of $g_{1}$, we obtain the desired equivariant bundle $\left(\pi, g_{1}^{*}(Q)\right)$ structure on $P$.

Note that at any point $p_{3} \in \operatorname{Fix}(X, \pi)$, with $p_{3} \neq p_{1}, p_{2}$, the rotation numbers of the pull-back equivariant bundle $\left(\pi, g_{1}^{*} Q\right)$ are the same as at the south pole $\left\{\left(-a_{1}+b_{1}\right),-\left(-a_{1}+b_{1}\right)\right\}$. Now the same pull-back-bundle construction can be repeated using an invariant 4-ball $B\left(p_{2}\right)$ around $p_{2}$, a corresponding degree-1 map $g_{2}: X \rightarrow S^{4}$, and the pull-back $\left(g_{2}\right)^{*}\left(Q^{\prime}\right)$ of an equivariant bundle $Q^{\prime}$ over $S^{4}$. This time, however, the rotation numbers of $\left(\pi, g_{2}^{*}\left(Q^{\prime}\right)\right)$ at $p_{3}$ are $\left\{\left(-a_{2}+b_{2}\right)\right.$, $\left.-\left(-a_{2}+b_{2}\right)\right\}$.

In order that there exists an arc $\gamma$ of self-dual equivariant connections connecting the 2 concentrated connections at $p_{1}$ and $p_{2}$, these 2 equivariant bundles $\left(\pi, g_{1}^{*}(Q)\right)$ and $\left(\pi, g_{2}^{*}\left(Q^{\prime}\right)\right)$ must be the same. Therefore the rotation numbers $\left\{\left(-a_{1}+b_{1}\right)\right.$, $\left.-\left(-a_{1}+b_{1}\right)\right\},\left\{\left(-a_{2}+b_{2}\right),-\left(-a_{2}+b_{2}\right)\right\}$ at $p_{3}$ must also agree. Since $\left(a_{1}, b_{1}\right)$ and $\left(a_{2}, b_{2}\right)$ represent a cancelling pair, it follows that either $2 a_{1} \equiv 0 \bmod |\pi|$ or $2 b_{1} \equiv 0 \bmod |\pi|$. This is impossible because $|\pi|$ is odd and the action $(\pi, X)$ has isolated fixed points at $p_{1}$ and $p_{2}$. 
We can now complete the proof of Theorem A, part 2 by considering the symmetries of the singular set patterns in $\bar{M}$ under the action of $\pi$. By the first part of Theorem $\mathrm{A}$, we can assume that $\pi \subset P G L_{3}(\mathbf{C})$ and that the action is a cone over some linear $\pi$-actions on $P^{2}(\mathbf{C})$ around each reducible connection.

To prove part 2 we refer to the list given in $[18, \S 1]$ for the various families of finite subgroups of $P G L_{3}(C)$. We must eliminate (i) nonabelian subgroups of $U(2)$, (ii) subgroups of the Hessian group, and (iii) the simple groups $A_{5}, A_{6}$, and $P S L_{2}\left(\mathbb{F}_{7}\right)$. These cases are handled by the following 3 lemmas: Lemma 18 for types (B)-(D), Lemma 19 for types (E)-(G), and Lemma 20 for types (H)-(J). Note that $A_{5}, A_{6}$ contain $A_{4}$, and $P S L_{2}\left(\mathbb{F}_{7}\right)$ contains a dihedral group of order 14 .

LEMMA 18. If $\pi$ contains a normal cyclic subgroup $\pi^{\prime}$ on which the conjugation action of $\pi$ is nontrivial and sends each element $g \mapsto g^{-1}$, for $g \in \pi^{\prime}$, then $\pi$ does not act smoothly and effectively on $X=\#_{1}^{n} P^{2}(\mathbf{C})$, inducing the identity on homology, for $n>1$.

Proof. The linear actions of $\pi$ on $P^{2}(\mathbf{C})$ restricted to the cyclic normal subgroup $\pi^{\prime}$ are of the form

$$
\left[\begin{array}{ccc}
1 & 0 & 0 \\
0 & \zeta^{a} & 0 \\
0 & 0 & \zeta^{-a}
\end{array}\right]
$$

where $a$ is relatively prime to the order of the subgroup. Therefore these actions have 3 isolated points for the $\pi^{\prime}$ action on the link of each reducible connection in $\overline{\mathcal{M}}$. It follows easily that at least 2 distinct reducibles must be connected by a 1-dimensional stratum of the fixed point set of $\pi^{\prime}$ intersecting the 2 linking copies of $P^{2}(\mathbf{C})$ at points $p$ and $q$, say. However, since this pattern has an extra $Z / 2$ symmetry, the rotation numbers at $p$ and $q$ must be of the form $(a,-a)$ and $(b,-b)$. Moreover, these rotation numbers at $p$ and $q$ must be identified under an orientation-reversing $\pi^{\prime}$-equivariant homeomorphism, and this is impossible since $a \neq 0$.

LEMMA 19. If $\pi=\mathbf{Z} / 3 \times \mathbf{Z} / 3$ acts smoothly on $X$ inducing the identity on homology, then the $\pi$-action on $\overline{\mathscr{M}}$ has 3 isolated fixed points on the link of each reducible connection.

Proof. The other possible pattern for the singular set of $\pi$ on $P^{2}(\mathbf{C})$ has 3 isolated fixed points for each $\mathbf{Z} / 3$ subgroup, but no fixed points for $\pi[18,2.2]$. Again we observe that at least 2 distinct reducible connections must be joined by a 1-dimensional fixed set for the $\mathbf{Z} / 3$-action. However, this pattern has no additional symmetry and so the $\pi$-action does not exist.

Lemma 20. The group $\pi=A_{4}$ does not act smoothly on $X$ inducing the identity on homology. 
Proof. We consider the singular set for the action of the subgroup $\mathbf{Z} / \mathbf{2} \times$ $\mathbf{Z} / \mathbf{2} \subset \pi$. By Lemma 17 we can conclude that at least 2 reducibles are again connected by a 1 -dimensional fixed set in $\overline{\mathscr{M}}$, and this pattern has no extra $\mathbf{Z} / 3$ symmetry.

Proof of Theorem $C$. First we suppose that $\pi=\mathbf{Z} / \mathbf{p}$ and consider the fixedpoint set $\operatorname{Fix}(\bar{M}, \pi)$, which contains $\operatorname{Fix}(X, \pi)$. By Theorem 15 we can assume that each reducible $[D]$ has an equivariant neighbourhood in $\mathscr{M}$ which is a cone over a linear $\pi$-action on $P^{2}(\mathbf{C})$. Each such cone has a fixed set consisting of either 3 distinct lines or a line and a cone over a 2-sphere. In addition, these components are disjoint except at the cone point $[D]$ and correspond to different isotropy subgroups of the $\pi \times S^{1}$ action in the local model.

Case (i). Assume that the $\mathbf{Z} / \mathbf{p}$-action has only isolated fixed points. Then by Theorem 16 the same is true for the linear actions linking the reducibles. We regard the reducibles $\left[D_{i}\right]$ and the distinct points $x_{j} \in \operatorname{Fix}(X, \pi)$ as the vertices of a graph. Two such vertices are connected by an edge if there is an equivalence class of paths in $\operatorname{Fix}(\bar{M}, \pi)$ connecting them. We consider such paths to be equivalent if they lie in the same component of Fix $(\overline{\mathscr{M}}, \pi)$ in every small open neighbourhood of the vertices in $\bar{M}$. The vertices $\left[D_{i}\right]$ have valence 3 (connected to exactly 3 others), and the vertices $\left\{x_{j}\right\}$ have valence 1 , by Lemma 17 . Since there are $n+2$ fixed points in $X$ and only $n$ reducibles, at least 1 reducible must be joined to 2 vertices in $X$.

On the other hand, if $n>1$ it is not possible for 1 reducible to be joined to 3 points in $X$, since then the remaining $n-1$ reducibles would be connected by $n-1$ edges. This would produce a closed path, say $\gamma$, of edges and $\gamma$ would intersect 1 of the cones in 2 lines $\ell_{1}, \ell_{2}$ of fixed points with distinct isotropy groups $\pi\left(\ell_{1}\right) \neq \pi\left(\ell_{2}\right)$ in the $\pi \times S^{1}$ action of the local model. But an irreducible $[D] \in \mathscr{B}^{\pi}$ corresponds to a lifting of the action on $X$ to a $\pi$-SU(2) bundle structure on $P$, and the $S^{1}$ determinant line bundles over the strata containing $\ell_{1}, \ell_{2}$ are both "positively oriented" by the complex structure on $H_{D}^{1}$. This contradicts the fact that the oriented determinant line bundle extends over $\gamma$, and shows that such a closed path $\gamma$ does not exist.

We can now count the edges and conclude that the reducibles may be ordered $\left[D_{1}\right], \ldots,\left[D_{n}\right]$ so that there is an edge $e(i, j)$ connecting $\left[D_{i}\right]$ and $\left[D_{j}\right]$ if and only if $j=i+1$, for $1 \leqslant i \leqslant(n-1)$. The remaining edges connect $\left[D_{1}\right]$ and $\left[D_{n}\right]$ to 2 fixed points each in $X$, and $\left[D_{i}\right], 2 \leqslant i \leqslant(n-1)$, to 1 fixed point each in $X$. This is exactly the pattern of the equivariant connected sum of linear actions. From this we can also conclude (from the normal bundles to Fix $\left(\overline{\mathscr{M}}^{*}, \pi\right)$ as usual) that the tangential representations at fixed points in $X$ are the same as those in an equivariant connected sum of linear actions.

Case (ii). When the $\mathbf{Z} / \mathbf{p}$ action has 2-dimensional components in $\operatorname{Fix}(X, \pi)$ the analysis is similar, but the definition of the graph must be modified. The vertices as before are the reducible connections together wth the components of 
$\operatorname{Fix}(X, \pi)$. The edges now have weights: $w(e)=1$ if the path lies in a 1-dimensional fixed-point set and $w(e)=2$ otherwise. Now the valence (the weighted sum of the edges) of each reducible is still 3. Consider one of the sets $S_{i}$ of reducibles given by Theorem B. If $k$ is the number of elements in $S_{i}$, and $n>k$, then 1 of the reducibles in $S_{i}$ must be joined by an edge of weight 1 to a reducible not in $S_{i}$. Otherwise, the $n-k$ remaining reducibles are joined by edges with weighted sum $n-k$, and a loop $\gamma$ would exist as before. The same contradiction eliminates this possibility, and we can conclude that the subgraph with vertices the reducibles is connected. An edge count shows that we again have the fixed-point pattern for an equivariant connected sum of linear actions. The tangential representations are determined by the normal bundles to the fixed sets as before.

Case (iii). Suppose now that $\pi$ is a cyclic group, and $\mathbf{Z} / \mathbf{p}$ is a subgroup of $\pi$. If the $\mathbf{Z} / \mathbf{p}$-action has isolated fixed points, then the $\pi$-action is pseudofree, and Theorem $\mathbf{C}$ follows in this case. If the $\mathbf{Z} / \mathbf{p}$-action has 2-dimensional fixed sets, the group $\pi$ acts on $\operatorname{Fix}(\overline{\mathscr{M}}, \mathbf{Z} / \mathbf{p})$ with fixed set $\operatorname{Fix}(\overline{\mathcal{M}}, \pi)$. Again, it follows that this fixed-point pattern is the same as that of an equivariant connected sum of linear actions, and the proof of Theorem $\mathrm{C}$ is complete.

6. A nonsmoothable action. In [11] a semifree action of $\pi=\mathrm{Z} / 5$ on $X=$ $P^{2}(\mathrm{C}) \# P^{2}(\mathrm{C})$ was constructed. Start with the linear actions on $P^{2}(\mathrm{C})$ having rotation numbers $\{(2,3),(3,1),(-1,2)\}$ and $\{(1,2),(-1,1),(-1,3)\}$. After the equivariant connected sum at the cancelling pair $(-1,2),(1,2)$, we obtain a smooth action on $X$ with rotation numbers

$$
\{(2,3),(3,1),(-1,1),(-1,3)\} \text {. }
$$

In particular, these values satisfy the Atiyah-Singer formula. Now, replacing the cancelling pair $(3,1),(-1,3)$ by the cancelling pair $(1,1),(1,-1)$, and applying Theorem 5 , the result is a topological, locally linear action with rotation numbers

$$
\{(2,3),(1,1),(-1,1),(1,-1)\}
$$

at the 4 isolated fixed points.

Such an action cannot arise as an equivariant connected sum of actions on $P^{2}(\mathrm{C})$ since the fixed-point data $(1,1)$ would imply the existence of 2 -spheres in the fixed-point set. Since the action induces the identity on homology, the Euler characteristic of the fixed point set is 4 , and so the action is pseudofree.

THEOREM 21. There is no smooth action of $\pi=Z / 5$ on $P^{2}(C) \# P^{2}(C)$ which induces the identity on homology and has isolated fixed points with tangential representations given by $\{(1,1),(1,4),(1,4),(2,3)\}$.

Proof. The equivariant moduli space $\mathscr{M}$ has 2 singular points $\left[D_{1}\right],\left[D_{2}\right]$ arising from reducible connections. By Lemma 17 , the 4 isolated fixed points in $X$ 
provide 4 1-dimensional fixed point sets in $\mathscr{M}$, and each of these has either $\left[D_{1}\right]$ or $\left[D_{2}\right]$ as its closure. By Theorem 15 , the moduli space is locally just the cone on some linear actions on $P^{2}(C)$ near the $\left[D_{i}\right]$. Since the fixed arcs from $X$ intersect the linking $P^{2}(C)$ in isolated fixed points, these linear actions have 3 isolated fixed points. However, the equivariant normal bundles to these fixed $\operatorname{arcs}$ in $\mathscr{M}$ are product bundles, and this implies that the slice representation $(1,1)$ would appear in a linear action on $P^{2}(C)$, a contradiction.

Remark 22. A. Edmonds has informed us that it is possible to produce a wide variety of rotation number configurations as the number of copies of $P^{2}(C)$ in the connected sum increases. The idea is to find "cancelling cyles" of rotation numbers, instead of just cancelling pairs. Combining this work with Theorem 5 and Theorem $\mathrm{C}$ would probably produce many other nonsmoothable locally linear actions of $\mathbf{Z} / \mathbf{p}$ on connected sums of $P^{2}(\mathbf{C})$.

\section{REFERENCES}

[1] M. F. AtiYAH AND I. M. Singer, The index of elliptic operators: III, Ann. of Math. (2) 87 (1968), 546-577.

[2] E. Bierstone, General position of equivariant maps, Trans. Amer. Math. Soc. 234 (1977), $447-$ 466.

[3] - "Generic equivariant maps" in Real and Complex Singularities, Sijthoff and Nordhoff, Alphen aan den Rijn, 1977, 127-161.

[4] G. E. Bredon, Introduction to Compact Transformation Groups, Academic Press, New York, 1972.

[5] Y. S. CHO, Finite group actions on the moduli space of self-dual connections (I), Trans. Amer. Math. Soc. 323 (1991), 233-261.

[6] - Finite group actions on the moduli space of self-dual connections (II), Michigan Math. J. 37 (1990), 125-132.

[7] S. K. DonaldSon, An application of gauge theory to 4-dimensional topology, J. Differential Geom. 18 (1983), 279-315.

[8] S. K. Donaldson And P. B. KronheImer, The Geometry of Four-Manifolds, Clarendon Press, Oxford, 1990.

[9] A. EDMONDS, Aspects of group actions on four-manifolds, Topology Appl. 31 (1989), 109-124.

[10] A. EDMONDS AND J. EWING, Locally linear group actions on the complex projective plane, Topology 28 (1989), 211-224.

[11] - Realizing forms and fixed-point data in dimension four, Amer. J. Math. 114 (1992), 1103-1126.

[12] D. S. Freed AND K. K. Uhlenbeck, Instantons and Four-Manifolds, Math. Sci. Res. Publ. 1, Springer-Verlag, New York, 1984.

[13] R. Fintushel AND T. LAwson, Compactness of moduli spaces for orbifold instantons, Topology Appl. 23 (1986), 305-312.

[14] R. Fintushel AND R. J. Stern, Pseudofree orbifolds, Ann. of Math. (2) 122 (1985), 335-364.

[15] I. HAMBLETON AND M. KRECK, Smooth structures on algebraic surfaces with cyclic fundamental group, Invent. Math. 91 (1988), 53-59.

[16] Cancellation of hyperbolic forms and topological four-manifolds, J. Reine Angew. Math. 443 (1993), 21-47.

[17] I. HAMBleton, R. LeE, AND I. MADSEN, Rigidity of certain finite group actions on the complex projective plane, Comment. Math. Helv. 64 (1989), 618-638.

[18] I. HAmbleton AND R. LeE, Finite group actions on $P^{2}(C)$, J. Algebra 116 (1988), 227-242. 
[19] - Perturbation of equivariant moduli spaces, Math. Ann. 293 (1992), 17-37.

[20] S. Illman, Subanalytic equivariant triangulation of real analytic proper G-actions, for $G$ a Lie group, preprint, Princeton Univ. 1992.

[21] T. LAwson, Compactness results for orbifold instantons, Math. Z. 200 (1988), 123-140.

[22] J. N. Mather, "Stratifications and mappings" in Dynamical Systems, ed. by M. Peixoto, Academic Press, New York, 1973, 195-232.

[23] D. M. WilczYNSKI, Group actions on the complex projective plane, Trans. Amer. Math. Soc. 303 (1987), 707-731.

Hambleton: Department of Mathematics, McMaster University, Hamilton, Ontario, CanADA, L8S 4K1

Lee: Department of Mathematics, Yale University, New Haven, Connecticut 06520, USA 